\title{
THE NUMBER OF ZERO SOLUTIONS FOR COMPLEX CANONICAL DIFFERENTIAL EQUATION OF SECOND ORDER WITH CONSTANT COEFFICIENTS IN THE FIRST QUADRANT
}

\author{
Jelena Vujaković ${ }^{*}$, Miloje Rajović \\ ${ }^{1}$ Faculty of Sciences and Mathematics, University of Priština, Kosovska Mitrovica, Serbia. \\ ${ }^{2}$ Faculty of Mechanical Engineering, University of Kragujevac, Kraljevo, Serbia.
}

\begin{abstract}
The study of complex differential equations in recent years has opened up some of questions concerning the determination of the frequency of zero solutions, the distribution of zero, oscillation of the solution, asymptotic behavior, rank growth and so on. Besides, this is solved by only some

classes of differential equations. In this paper, our aim was to determine the number of zeros and their arrangement in the first quadrant, for the complex canonical differential equation of the second order. The accuracy of our results, we illustrate with two examples.
\end{abstract}

Key words: Differential equations, function of frequency, sine solution, cosine solution, zero solutions.

\section{INTRODUCTION}

The study of complex differential equations, in terms Nevanlinna theory, becomes actual again since 1982, the publication of the following works: Bank (1988), (Bank \& Laine, 1982; 1983), (Bank et al., 1989).

Then are generally treated canonical complex differential equations of second order with a coefficient which is an entire function. All of these studies have focused mainly on two general issues.

The first one involved the determination of the frequency of zero solutions, while the other studied the distribution and the asymptotic behavior of zero solutions in the first quadrant i.e., in the sector $0 \leq \varphi \leq \frac{\pi}{2},|z|=R$. About the problem of distribution of zero solutions of complex differential equations, the case where the coefficient $a(z)$ is polynomial $P_{n}(z)$ is quite clear. When $a(z)$ is the transcendent function the situation is much more complex. Review of the scientific literature, such as (Gundersen, 1986), (Laine, 1993), (Shu Pei, 1994) and others, shows that there are mostly treated complex differential equations with transcendental coefficients $e^{z}$ and coefficients derived from it: $e^{z}+P_{n}(z), e^{z} \cdot P_{n}(z), P_{n}\left(e^{z}\right), \ldots \ldots$ This is because in Nevanlinna theory as a measure of transcendence and infinite growth, takes the function $e^{z}$. Since $\left|e^{z}\right|=e^{x}, x \in \mathbb{R}$, as $x \rightarrow \infty$ function $e^{z}$ tends to complex infinity of transcendent type.

Unlike classical Nevanlinna theory, we are using the the idea of (Dimitrovski \& Mijatović, 1998), (Lekić et. al., 2012), (Vujaković et al., 2011), (Vujaković, 2012) developed a new approach in determining the location and number of zero solutions. This method looked better in the applications for us.

In this paper, the subject of our considerations is complex canonical differential equations of second order with constant coefficients.

\section{PRELIMINARIES}

For complex canonical differential equation of the second order :

$$
\frac{d^{2} w}{d z^{2}}+a(z) w(z)=0
$$

with an analytical coefficient $a(z)=\alpha(x, y)+i \beta(x, y), \quad$ where $\alpha(x, y)$ and $\beta(x, y)$ are harmonic functions, by series-iterations method which are described in detail in the works (Dimitrovski \& Mijatović, 1998), (Lekić et al., 2012), (Vujaković, 2012), we get two fundamental solutions: 


$$
\begin{aligned}
& w_{1}(z)=\cos _{a(z)} z \\
& =1-\int_{0}^{z} \int_{0}^{z} a(z) d z^{2}+\int_{0}^{z} \int_{0}^{z} a(z) d z^{2} \int_{0}^{z} \int_{0}^{z} a(z) d z^{2}-\ldots \approx \\
& \approx \cos (z \sqrt{a(z)}), \\
& w_{2}(z)=\sin _{a(z)} z \\
& =\frac{1}{\sqrt{a(z)}}\left[z-\int_{0}^{z} \int_{0}^{z} z a(z) d z^{2}+\int_{0}^{z} \int_{0}^{z} a(z) d z^{2} \int_{0}^{z} \int_{0}^{z} z a(z) d z^{2}-\ldots\right] \\
& \approx \frac{\sin (z \sqrt{a(z)})}{\sqrt{a(z)}} \text {. }
\end{aligned}
$$

Function $w_{1}(z)$ and $w_{2}(z)$ we called oscillatory complex functions with base $a(z)$. Mark them with (2) and (3), respectively. Further, let

$$
F(z)=z \sqrt{a(z)}
$$

denotes a function of the frequency. We have seen in the papers (Vujaković et al., 2011; 2016), (Vujaković, 2012) that the zero solutions (2) and (3) are approximately in the solutions of equations

$$
\left.\begin{array}{l}
w_{1}: z \sqrt{a(z)}=(2 n-1) \frac{\pi}{2}, n=1,2,3, \ldots \\
w_{2}: z \sqrt{a(z)}=n \pi, n=0,1,2, \ldots
\end{array}\right\} .
$$

Therefore, it is important to know the behavior of $\sqrt{a(z)}$ for the analytical functions $a(z)=\alpha(x, y)+i \beta(x, y)$.

Theorem 1. For analytic function $a(z)=\alpha(x, y)+i \beta(x, y), \quad$ in complex canonical second-order differential equation (1), it is possible to determine the equation of zero cosine and sine solutions.

Proof. Putting $z=x+i y \quad$ and $a(z)=\alpha(x, y)+i \beta(x, y)$, for example in the second equation of the system (5), after the elementary calculation (i.e., squaring, multiplying complex numbers and on the basis of equality of complex numbers), we obtain the following system of equations for $\alpha(x, y)$ and $\beta(x, y)$.

$$
\begin{aligned}
& \left(x^{2}-y^{2}\right) \alpha(x, y)-2 x y \beta(x, y)=n^{2} \pi^{2} \\
& \left(x^{2}-y^{2}\right) \beta(x, y)+2 x y \alpha(x, y)=0 .
\end{aligned}
$$

Elimination $\beta(x, y)$ from the second equation of the last system and substituting them in the first equation of the same system we get the equation for $\alpha(x, y)$. We got the system of equations

$$
\left.\begin{array}{l}
\alpha(x, y)=n^{2} \pi^{2} \frac{x^{2}-y^{2}}{\left(x^{2}+y^{2}\right)^{2}}, \\
\beta(x, y)=-n^{2} \pi^{2} \frac{2 x y}{\left(x^{2}+y^{2}\right)^{2}}
\end{array}\right\}
$$

If $\alpha(x, y)$ and $\beta(x, y)$ are given, that is, if known coefficient $a(z)$, from (6) we find the zero $\left(x_{n}, y_{n}\right)$ of sine solutions $w_{2}(z)$ of complex differential equations of the second order (1). This means that (6) is a system of biquadratic equations. From (6), dividing $\alpha(x, y)$ and $\beta(x, y)$, we get

$$
\frac{x^{2}-y^{2}}{2 x y}=-\frac{\alpha(x, y)}{\beta(x, y)} .
$$

This equation is easy to solve only for the constant $a(z)=c_{1}+i c_{2}$.

Zero of cosine solutions for the canonical complex differential equations of the second order (1) can be found in a similar manner. Namely, from the first equation of the system (5) we have $(x+i y) \sqrt{\alpha(x, y)+i \beta(x, y)}=(2 n-1) \frac{\pi}{2}, n=1,2,3, \ldots$. From here, similarly as moment ago, we obtain a system of equations

$$
\left.\begin{array}{l}
\alpha(x, y)=\left(n-\frac{1}{2}\right)^{2} \pi^{2} \frac{x^{2}-y^{2}}{\left(x^{2}+y^{2}\right)^{2}}, \\
\beta(x, y)=-\left(n-\frac{1}{2}\right)^{2} \pi^{2} \frac{2 x y}{\left(x^{2}+y^{2}\right)^{2}} .
\end{array}\right\}
$$

Solving the system (5) is not a trivial task for all forms of the coefficient $a(z)=\alpha(x, y)+i \beta(x, y)$. The best and easiest way is if you have zero solutions of . $w_{1}(z)=\cos (z \sqrt{a(z)})$ and $w_{2}(z)=\sin (z \sqrt{a(z)})$ at a proper geometric line, so there is some law of their schedule.

Theorem 2. Zeros solutions for $w_{1}(z)=\cos \lambda z$ (and similar to $\left.w_{2}(z)=\sin \lambda z\right)$ exists if a module of the function $a(z)$ is right proportional to the order of

\section{Mathematics}


$\left(n-\frac{1}{2}\right)^{2}$ zero (respectively $n^{2}$ ) and inversely proportional to the square of the module $\rho$.

Proof. Using the polar form $z=\rho e^{i \varphi}$ of a complex number, for example, from the system (7), we obtain the system

$$
\left.\begin{array}{l}
\alpha=\alpha(\rho, \varphi)=\left(n-\frac{1}{2}\right)^{2} \pi^{2} \frac{\cos 2 \varphi}{\rho^{2}}, \\
\beta=\beta(\rho, \varphi)=-\left(n-\frac{1}{2}\right)^{2} \pi^{2} \frac{\sin 2 \varphi}{\rho^{2}}
\end{array}\right\}
$$

From here we have $\alpha^{2}+\beta^{2}=\left(n-\frac{1}{2}\right)^{4} \frac{\pi^{4}}{\rho^{2}}$, that is we obtain the connection between the $\alpha$ and $\beta$, which does not depend on the argument $2 \varphi$. If we write $a(z)$ in polar form $a(z)=\alpha(x, y)+i \beta(x, y)=R e^{i \theta}$, we have $R=\left(n-\frac{1}{2}\right)^{2} \frac{\pi^{2}}{\rho^{2}}$. Besides, from $\tan \theta=\frac{\beta}{\alpha}$ follows $\tan \theta=-\tan 2 \varphi=\tan (-2 \varphi)$, or equivalent $\theta=-2 \varphi+k \pi$.

By the same reasoning from the system (6) we prove the assertion for $w_{2}(z)=\sin \lambda z$.

\section{MAIN RESULTS}

For starters, it is important to determine the number of zeros solutions of complex second order differential equation (1) for a constant $a(z)=c_{1}+i c_{2}$, or to try to evaluate zeros better. Because of the multifaceted analytic functions it is best first to observe the characteristic examples of differential equations with constant coefficients

$$
\frac{d^{2} w}{d z^{2}}+a(z) w(z)=0, a(z)=c_{1}+i c_{2}
$$

The problem is that we must know in advance for which $a(z)$, the value of $\sqrt{a(z)}=\sqrt{c_{1}+i c_{2}}$, which is ambiguous, it remains in the first quadrant, and when he leaves. Therefore, consider the four basic equations.

1. Assume first that $a(z)=1$, is pure real constant coefficient. Follows, $c_{1}=1>0, c_{2}=0$. Then $\sqrt{a(z)}= \pm 1$ and a function of frequency is $z \sqrt{a(z)}= \pm z$. The solutions of the canonical complex differential equation of the second order $\frac{d^{2} w}{d z^{2}}+w(z)=0 \quad$ are $\quad w_{1}(z)=\cos z, w_{2}(z)=\sin z$. Zeros of sine solutions $z=n \pi, n=0,1,2, \ldots$ are on the real axis (limit of the first quadrant).

2. For real constant coefficient $a(z)=-1$ we have $c_{1}=-1<0, c_{2}=0$. Then $\sqrt{a(z)}=\sqrt{-1}= \pm i$, and $z \sqrt{a(z)}= \pm i z$. The solutions of canonical complex differential equations of the second order $\frac{d^{2} w}{d z^{2}}-w(z)=0 \quad$ are $\quad w_{1}(z)=\cos (i z) \quad$ and $w_{2}(z)=\sin (i z)$. Zeros of sine solutions are in the solutions of equations $z=-n \pi, n=0,1,2, \ldots$. They are on the imaginary axis and not in the first quadrant, except solution $z=0$.

3. Consider now the complex canonical secondorder differential equation $\frac{d^{2} w}{d z^{2}}+i w(z)=0$, with the imaginary constant coefficient $a(z)=i$, where $c_{1}=0, c_{2}=1>0$. Then $\sqrt{a(z)}=\sqrt{i}= \pm \frac{1+i}{\sqrt{2}}$, and a function of frequency is $z \sqrt{a(z)}=z\left( \pm \frac{1+i}{\sqrt{2}}\right)$. Solutions of complex differential equations are $w_{1}(z)=\cos \left( \pm \frac{i+1}{\sqrt{2}} z\right)$ and $w_{2}(z)=\sin \left( \pm \frac{i+1}{\sqrt{2}} z\right)$. Zeros of sine solutions $z= \pm(1-i) \frac{n \pi}{\sqrt{2}}, n=0,1,2, \ldots$ are in the second or fourth quadrant. So, there is no zero in the first quadrant.

For complex canonical second-order differential equation $\frac{d^{2} w}{d z^{2}}-i w(z)=0$, with the imaginary constant coefficient $a(z)=-i$, are $c_{1}=0, c_{2}=-1<0$ and $\sqrt{a(z)}=\sqrt{-i}= \pm \frac{1-i}{\sqrt{2}}$. Zeros of sine solutions are $z= \pm(1+i) \frac{n \pi}{\sqrt{2}}, n=0,1,2, \ldots$. From this, we conclude that there are zeros in the first and third quadrant.

By this elementary examination, we find out that only if $\operatorname{Im} a(z)=\operatorname{Im}\left(c_{1}+i c_{2}\right)<0$, is likely to be zeros of sine solutions in the first quadrant. However, we need a more general and more secure approach to solving this problem, but not elementary examples.

\section{Mathematics}


Theorem 3. For complex canonical second-order differential equation (9), with constant coefficients $a(z)=c_{1}+i c_{2}$, sine solution has zero in the first quadrant if $c_{2}<0$.

Proof. For a constant $a(z)=c_{1}+i c_{2}, \quad z=x+i y$, zeros of sine solutions are in the roots of the equations $z \sqrt{a(z)}=n \pi, n=0,1,2, \ldots$. Hence, according to the formula (6), we obtain the system:

$$
\left.\begin{array}{l}
c_{1}=n^{2} \pi^{2} \frac{x^{2}-y^{2}}{\left(x^{2}+y^{2}\right)^{2}}, \\
c_{2}=-n^{2} \pi^{2} \frac{2 x y}{\left(x^{2}+y^{2}\right)^{2}} .
\end{array}\right\}
$$

Because we want that $z=x+i y$ be in the first quadrant, therefore that $x>0, y>0$ holds, it must be $c_{2}<0$ in the $a(z)=c_{1}+i c_{2}$. If this holds yet for $x>y$ then $c_{1}>0$. In case $x<y$ we have that $c_{1}<0$.

Let us now return to solving the system of equations (10). By switching to polar form $z=\rho e^{i \varphi}$, from $c_{1}=n^{2} \pi^{2} \frac{\cos 2 \varphi}{\rho^{2}}$ and $c_{2}=-n^{2} \pi^{2} \frac{\sin 2 \varphi}{\rho^{2}}$, we find $\varphi=-\frac{1}{2} \arctan \frac{c_{2}}{c_{1}}$. Now, from $c_{1}=n^{2} \pi^{2} \frac{\cos 2 \varphi}{\rho^{2}}$ following $\rho=\frac{n \pi}{\sqrt[4]{c_{1}^{2}+c_{2}^{2}}}$. Substituting these values in $x=\rho \cos \varphi$ and $y=\rho \sin \varphi$ respectively, after the well-known trigonometric identities for the sine and cosine, we get the coordinates for the zeros of sine solutions of the complex canonical differential equations of the second order:

$$
\begin{gathered}
x=x(n)=\frac{n \pi}{\sqrt[4]{c_{1}^{2}+c_{2}^{2}}} \sqrt{\frac{\sqrt{c_{1}^{2}+c_{2}^{2}}+c_{1}}{2 \sqrt{c_{1}^{2}+c_{2}^{2}}}}, \\
y=y(n)=-\frac{n \pi}{\sqrt[4]{c_{1}^{2}+c_{2}^{2}}} \sqrt{\frac{\sqrt{c_{1}^{2}+c_{2}^{2}}-c_{1}}{2 \sqrt{c_{1}^{2}+c_{2}^{2}}}},
\end{gathered}
$$

for $n=0,1,2, \ldots$

From equations (12) it is evident that $y<0$, and comparing equations (11) with (12) we see that $|x|>|y|$. Dividing the (12) with (11) we obtain the

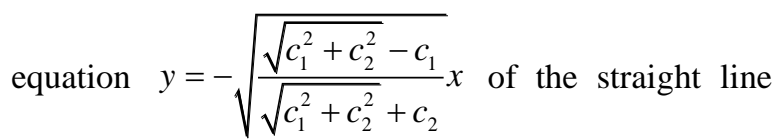
through the second and fourth quadrant.

We still need to determine how many zeros have a sine i.e., cosine solution of canonical complex differential equations of second order in the sector $|z|<R, 0 \leq \arg z<\frac{\pi}{2}$.

Theorem 4. In canonical complex second-order differential equation (9), with constant coefficients, the number of zeros of sine solutions is determined by the formula $N\left(z_{k}\right)=k E\left[\frac{\pi}{\sqrt[4]{\alpha^{2}+\beta^{2}}}\right]$, where $E$ is a whole part of the above argument, and $k$ is an integer unit in the $|z|<R, 0 \leq \arg z<\frac{\pi}{2}$. Cosine solution has one less zero.

Proof. For complex canonical differential equation of second order

$$
\frac{d^{2} w}{d z^{2}}+(\alpha+i \beta) w(z)=0
$$

with constant coefficients_ $a(z)=\alpha+i \beta$, according to Theorem 3 , it follows that zeros of sine solutions do not exist for all $\alpha, \beta$, but only for those values $\alpha=c_{1}, \beta=c_{2}$, that satisfy equations (11) and (12). Then we get the points $z_{n}=x_{n}+i y_{n}$ of region $|z|<R, 0 \leq \arg z<\frac{\pi}{2}$.

$\begin{array}{cccr}\text { From } \quad \text { a } \quad \text { function } & \text { of } & \text { frequency } \\ z \sqrt{a(z)}=n \pi, n=0,1,2, \ldots & \text { we } & \text { have }\end{array}$
$z_{n}=\frac{n \pi}{\sqrt[4]{\alpha^{2}+\beta^{2}}}, n=0,1,2, \ldots$. We see that this is a series of zeros per the length $\left|z_{n}\right|=\frac{n \pi}{\sqrt[4]{\alpha^{2}+\beta^{2}}}$ of some direction, which runs from $z=0$ to a point on a circle of radius $R$. The number of zeros is the number of slices on a circle of $R$ and has a lenght $\frac{n \pi}{\sqrt[4]{\alpha^{2}+\beta^{2}}}$.

If we introduce the function $E(x)=[x]$ (the whole part of the argument $x$ ), based on the properties $E[n r]=n E[r]$, where $n$ is a positive integer, we have for the number of zeros:

\section{Mathematics}




$$
N\left(z_{n}\right)=E\left[\frac{n \pi}{\sqrt[4]{\alpha^{2}+\beta^{2}}}\right]=n E\left[\frac{\pi}{\sqrt[4]{\alpha^{2}+\beta^{2}}}\right]
$$

Notice that the sine zeros are equidistant because for two consecutive $n$ and $n+1$ is valid

$$
\begin{aligned}
& \left|N\left(z_{n+1}\right)-N\left(z_{n}\right)\right|= \\
& =\left|(n+1) E\left[\frac{n \pi}{\sqrt[4]{\alpha^{2}+\beta^{2}}}\right]-n E\left[\frac{n \pi}{\sqrt[4]{\alpha^{2}+\beta^{2}}}\right]\right| \\
& =E\left[\frac{\pi}{\sqrt[4]{\alpha^{2}+\beta^{2}}}\right]
\end{aligned}
$$

Concerning the property that the sine function within a period $2 \pi$ has three zeros $0, \pi, 2 \pi$ and the cosine function in the same period has only two zeros $\frac{\pi}{2}, \frac{3 \pi}{2}$, we conclude that the cosine solution has one less zero than sine.

\section{EXAMPLES}

Let us illustrate the validity of our results in the examples.

Example 1. Sine solution of canonical complex differential equations of second order $\frac{d^{2} w}{d z^{2}}+(2-i) w(z)=0$, with constant coefficients $a(z)=2-i$, for which are $c_{2}<0$ and $\left|c_{1}\right|>\left|c_{2}\right|$, has a zero in the sector $|z|<R, 0 \leq \arg z<\frac{\pi}{2}$. Number of zeros will be equal:

$$
\begin{aligned}
E\left[\frac{n \pi}{\sqrt[4]{2^{2}+(-1)^{2}}}\right] & =n E\left[\frac{\pi}{\sqrt[4]{5}}\right]=n E\left[\frac{3,14}{1,5}\right]= \\
& =n E[2,1]=2 n .
\end{aligned}
$$

For example, if $R=10$, then from $2 n=10$ follows that there are $n=5$ sine zeros.

Example 2. Let were given complex canonical second-order differential equations $\frac{d^{2} w}{d z^{2}}+(3-2 i) w(z)=0 \quad$ with constant coefficient $a(z)=3-2 i$. Here are $c_{1}=3, c_{2}=-2$, so the conditions $c_{2}<0$ and $\left|c_{1}\right|>\left|c_{2}\right|$ are fulfilled. Sine solution is in the circle. For example, in circle of radius $|z|=R=100$ there are 50 zeros because:

$$
\begin{aligned}
N\left(\sin _{(3-2 i)} z\right) & =E\left[\frac{n \pi}{\sqrt[4]{3^{2}+(-2)^{2}}}\right]=n E\left[\frac{\pi}{\sqrt[4]{13}}\right]= \\
& =2 n=100
\end{aligned}
$$

\section{ACKNOWLEDGMENT}

Financial support for this study was granted by the Ministry of Education, Science and Technology Development of the Republic of Serbia (Project Number TR 35030).

\section{REFERENCES}

Bank S., 1988. On determining the location of complex zeros of solutions of certain linear differential equations, Annali di Matematica Pura ed Applicata, $\quad 151(1), \quad$ pp. 67-96. DOI:10.1007/BF01762788

Bank S., \& Laine I., 1982. On the oscillation theory of $f^{\prime \prime}+A f=0$ where $A$ is entire, Transactions of the American Mathematical Society, 273(1), pp. 351363.

Bank S., \& Laine I., 1983. On the zeros of meromorphic solutions of second order linear differential equations, Commentarii Mathematici Helvetici, 58, pp. 656-677.

Bank S., Laine I., \& Langley K., 1989. Oscillation results for solutions of linear differential equations in the complex domain, Results in Mathematics, 16, pp. 3-15.

Dimitrovski D., \& Mijatović M., 1998. A seriesIteration Method in the Theory of Ordinary Differential Equations, Hadronic Press.

Gundersen G., 1986. On the real zeros of solutions of $f^{\prime \prime}+A(z) f=0$ where $A(z)$ is entire, Annales Academiae Scientiarum Fennicae Mathematica, 11, pp. 275-294.

Laine I. 1993, Nevanlinna theory and complex differential equations, de Gruyter Studies in Mathematics 15, Walter de Gruyter, Berlin/New York 1993.

Lekić M., Cvejić S., \& Dimitrovski D., 2012. Sturm theorems through iteration, Monography, University of Priština, Faculty of Sciences and Mathematics, Kosovska Mitrovica, (in Serbian).

Shu Pei W., 1994. On the sectorial oscillation theory of $f^{\prime \prime}+A(z) f=0, \quad$ Annales Academiae Scientiarum Fennicae, Helsinki.

Vujaković J., Rajović M., \& Dimitrovski D. 2011. Some new results on a linear equation of the second order, Computers and Mathematics with Applications 61, pp. 1837-1843, doi: 10.1016/j.camwa.2011.02.012.

Vujaković J., 2012. Zeros solution of complex differential equations, Dissertation, University of 
Priština, Faculty of Sciences and Mathematics, Kosovska Mitrovica, (in Serbian)

Vujaković J., Lalović Lj., \& Rajović M., 2016. Equation of oscillations with one common cause of oscillations and with variable amplitudes, Journal of

${ }^{*}$ E-mail: jelena.vujakovic@pr.ac.rs
Advanced Mathematical Studies Bucharest, Vol. 9(2), pp.259-265,

http://journal.fairpartners.ro/volume-92016-no-2_17.html, Article in Press. 